\title{
Latest Developments in the New Wavelength Dispersive Solid-State Detector
}

\author{
D.P. Lesher, ${ }^{*}$ Y. Diawara, ${ }^{* *}$ S. Medved, ${ }^{* *}$ R. Durst, ${ }^{* *}$ Vladislav Sedov** \\ * Advanced MicroBeam, Inc., 4217C King Graves Rd., Vienna, OH 44473 USA \\ ** Bruker AXS Inc., 5465 East Cheryl Parkway, Madison, WI 53711-5373 USA
}

During the Microscopy and Microanalysis 2008 conference in Albuquerque, we discussed our initial experiments with the solid-state detector for Wavelength Dispersive spectrometers. Count rates approached 10 million counts per second and were limited by the counting capacity of the first generation electronics. The initial system was capable of detecting down to oxygen without the use of additional cooling. A cooled solid-state detector has been developed that is capable of counting $\mathrm{Be}, \mathrm{B}, \mathrm{C}, \mathrm{N}$, and $\mathrm{O}$ x-rays. Count rate tests will show how the detector performs for these elements. For light currents (1 to 500 nanoamps.), and medium currents (500 to 5000 nanoamps.), the solidstate detector provided the following advantages:

* 25 to 50 times faster pulse processing times than typical x-ray electronics that produce 1 to 2 microsecond pulse processing times

* Minimal software dead time corrections are required - improving accuracy

* Similar or better quantum efficiency than a xenon proportional counter

* No detector saturation and no false x-ray maps

* Simpler manufacturing process when compared to a proportional counter

The latest second generation circuit improvements have increased the counting capacity. The solidstate detector is capable of up to 50 million counts per second, and experiments show that the new circuitry can achieve 25 million counts per second (Figure 1). This circuitry produces shorter, well defined amplifier pulses, reduces baseline shifts due to count rates, takes advantage of high-speed de-randomizer circuits to improve processing times, and has an improved discriminator for setting baselines and windows. 


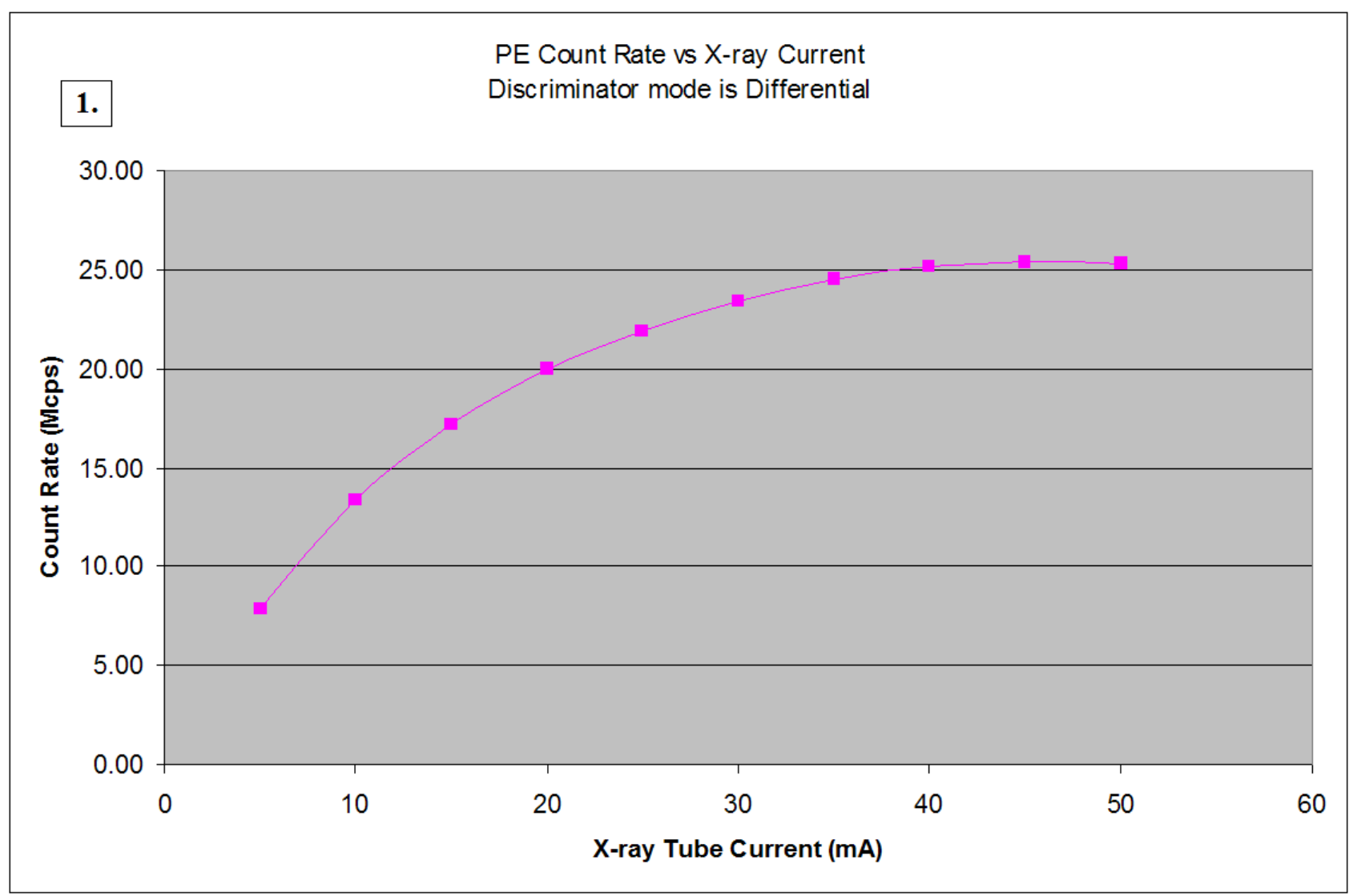

Fig. 1. Copper Count Rates - 20KV - Using Solid State Detector with $2^{\text {nd }}$ Generation Circuitry 